\title{
Roles of Type VI Secretion System in Transport of Metal Ions
}

\author{
Xiaobing Yang ${ }^{1,2}$, Hai Liu ${ }^{3 *}$, Yanxiong Zhang ${ }^{3}$ and Xihui Shen ${ }^{4 *}$ \\ ${ }^{1}$ College of Applied Engineering, Henan University of Science and Technology (HAUST), Sanmenxia, China, ${ }^{2}$ Medical \\ College, Sanmenxia Vocational Technical School, Sanmenxia, China, ${ }^{3}$ Qingyang Longfeng Sponge City Construction \\ Management \& Operation Co., Ltd, Qingyang, China, ${ }^{4}$ State Key Laboratory of Crop Stress Biology for Arid Areas, Shaanxi \\ Key Laboratory of Agricultural and Environmental Microbiology, College of Life Sciences, Northwest A\&F University, \\ Xianyang, China
}

The type $\mathrm{VI}$ secretion system (T6SS) is a transmembrane protein nanomachine employed by many gram-negative bacteria to directly translocate effectors into adjacent cells or the extracellular milieu, showing multiple functions in both interbacterial competition and bacteria-host interactions. Metal ion transport is a newly discovered T6SS function. This review summarizes the identified T6SS functions and highlights the features of metal ion transport mediated by T6SS and discusses its regulation.

OPEN ACCESS

Edited by:

Eric Cascales,

Aix-Marseille Université, France

Reviewed by:

Miguel A. Valvano,

Queen's University Belfast,

United Kingdom

Jin $\mathrm{He}$,

Huazhong Agricultural University,

China

*Correspondence: Hai Liu

liuhai@capitalwater.cn Xihui Shen

xihuishen@nwsuaf.edu.cn

Specialty section:

This article was submitted to Microbial Physiology and Metabolism,

a section of the journal

Frontiers in Microbiology

Received: 10 August 2021

Accepted: 12 October 2021

Published: 05 November 2021

Citation:

Yang $X$, Liu H, Zhang $Y$ and Shen X (2021) Roles of Type VI

Secretion System in Transport

of Metal lons.

Front. Microbiol. 12:756136

doi: 10.3389/fmicb.2021.756136
Keywords: type VI secretion system, effectors, metal ions, transport, regulation

\section{INTRODUCTION}

The type VI secretion system (T6SS) is a transmembrane protein nanomachine employed by many gram-negative bacteria to translocate effectors directly into adjacent target cells or the extracellular milieu (Cianfanelli et al., 2016). T6SS was regarded as virulence-associated secretion apparatus because of its association with pathogenicity (Mougous et al., 2006; Pukatzki et al., 2006). However, subsequent studies have demonstrated T6SS function is involved in multiple physiological and biochemical processes apart from bacterial pathogenesis, such as interbacterial competition (Hood et al., 2010; Chassaing and Cascales, 2018), commensalism or symbiosis (Chow and Mazmanian, 2010), stress response (Weber et al., 2009; Wan et al., 2017), biofilm formation (Zhang et al., 2011; Gallique et al., 2017), and horizontal gene transfer (Borgeaud et al., 2015).

It has been reported that the T6SS function is determined by the loading effectors that can be delivered extracellularly based on energy consumption and load transport (Cianfanelli et al., 2016; Coulthurst, 2019). Many T6SS effectors related to virulence or competition that target the cell wall, membranes, and nucleic acids have been reported (Yang et al., 2018; Song et al., 2021). Several effectors with special activities have also been found. For example, a T6SS dependent effector, YezP, has been reported to combine with $\mathrm{Zn}^{2+}$ and contribute to $\mathrm{Zn}^{2+}$ transport to deal with environmental stresses (Wang et al., 2015), and subsequent studies confirmed the function of T6SS dependent transport of metal ions (Lin et al., 2017; Si et al., 2017b). This review highlights the features of T6SS-dependent metal ion transport and its regulation.

\section{TYPE VI SECRETION SYSTEM FUNCTIONS FOR METAL IONS TRANSPORT}

Metal ions are commonly found in all organisms in association with proteins, such as enzymes, storage proteins, and transcription factors. The metal ions are involved in many crucial biological

Abbreviations: T6SS, type VI secretion system; Azu, azurin. 
processes and are necessary for cell survival (Hood and Skaar, 2012). Bacteria have evolved sophisticated acquisition systems, including low- and high-affinity transport systems for scavenging essential chelated or free metals from the environment (Porcheron et al., 2013). As a versatile secretion system widely distributed in Gram-negative bacteria, The T6SS was found to participate in the transport of iron, zinc, copper, manganese, and molybdate, summarized in Table $\mathbf{1 .}$

\section{Zinc}

Zinc is the second most important transition metal ion in living organisms after iron, playing an essential catalytic and structural role in several proteins involved in DNA replication, glycolysis, $\mathrm{pH}$ regulation, amino acid biosynthesis, extracellular peptidoglycan, and low molecular weight thiols (Porcheron et al., 2013). Zinc status is linked to the maintenance of intracellular redox buffering (Andreini et al., 2006). Both the high-affinity transporter ZnuACB and the low-affinity uptake system ZupT mediate zinc uptake across the cytoplasmic membrane (Hantke, 2005). Zinc is an essential nutrient for cells; Excess of zinc is toxic. Therefore, bacterial cells should achieve a delicate balance between ensuring sufficient zinc concentrations to fulfill essential functions while limiting concentration to prevent toxic effects. Zinc homeostasis is mediated by a network of zinc influx and efflux pumps (Wang et al., 2012; Wang and Fierke, 2013).

Wang et al. (2015) reported that the T6SS-4 from Yersinia pseudotuberculosis (Yptb) can combat multiple adverse stresses and host nutritional immunity, by displaying an unexpected function in transport of $\mathrm{Zn}^{2+}$. Zinc transport is achieved by T6SS-4-mediated secretion of a $\mathrm{Zn}^{2+}$-binding protein substrate, YezP (YPK_3549), which binds $\mathrm{Zn}^{2+}$ with high affinity, and represents a novel class of T6SS effector distinct from those extensively studied as bacteriolytic toxins or eukaryotic celltargeting effectors. Hydroxyl radicals are deleterious reactive oxygen species that are often generated via Fenton chemistry under stress conditions (Mols and Abee, 2011). T6SS-4 was critical to neutralize hydroxyl radicals accumulated under adverse stress conditions, by accumulating $\mathrm{Zn}^{2+}$, which can mitigate hydroxyl radicals to reduce them damage. By mitigating the detrimental hydroxyl radicals induced by multiple stresses, T6SS-4 provided a molecular explanation to the phenomenon of

TABLE 1 | T6SS dependent ions transport related factors.

\begin{tabular}{lclll}
\hline Bacteria species & $\begin{array}{c}\text { T6SS } \\
\text { effector }\end{array}$ & $\begin{array}{l}\text { Membrane } \\
\text { transporter }\end{array}$ & Metal ions & Citation \\
\hline Y. pseudotuberculosis & YezP & & Zinc & Wang et al., 2015 \\
Y. pseudotuberculosis & TssS & & Manganese & Zhu et al., 2021 \\
B. thailandensis & TseM & MnoT & Manganese & Si et al., 2017b \\
B. thailandensis & TseZ & HmuR & Zinc & Si et al., 2017a \\
B. pseudomallei & TseZ & BhuR & Zinc & DeShazer, 2019 \\
B. pseudomallei & TseM & MnoT & Manganese & DeShazer, 2019 \\
P. aeruginosa & TseF & FptA/OprF & Iron & Lin et al., 2017 \\
P. aeruginosa & Azu & OprC & Copper & Han et al., 2019 \\
P. aeruginosa & ModA & IcmP & Molybdate & Wang et al., 2021 \\
C. necator & TeoL & CubA/CstR & Iron & Li et al., 2021
\end{tabular}

"cross-protection" in which cells subjected to one stress become resistant to distinctly different insults (Isohanni et al., 2013). Consistent with the function of T6SS-4 in combating stress, its expression is regulated by multiple transcription regulators, such as OmpR (Gueguen et al., 2013; Zhang et al., 2013), OxyR (Wang et al., 2015), RovM (Song et al., 2015), RpoS (Guan et al., 2015), and RelA (Yang et al., 2019), all of which respond to various stresses (Song et al., 2015; Zhao et al., 2017; Yang et al., 2019). Both ZntR and Zur, two zinc responsive regulators, are also involved in T6SS-4 regulation by directly binding to its promoter region (Wang et al., 2017; Cai et al., 2021).

The type VI secretion system-4 dependent zinc transport also plays a crucial role in the interactions of pathogenic $Y$. pseudotuberculosis with its mammalian host, as Y. pseudotuberculosis T6SS-4 mutants are attenuated in virulence against mice. Especially, mutation of T6SS-4 or yezP together with $z n u C B$ [a classic zinc transporter known to combat host nutritional immunity (Hood et al., 2012; Liu et al., 2012)] resulted in mutants that almost completely lost the virulence against mice, suggesting the importance of T6SS-4 the resistance to host nutritional immunity (Wang et al., 2015). This finding revealed a new mechanism of T6SS in pathogenesis. Further studies on Burkholderia thailandensis have revealed the mechanism of zinc ions transport across the membrane through T6SS (Si et al., 2017a). The T6SS-4 in B. thailandensis is involved in zinc acquisition via contact-independent secretion of a zinc-scavenging protein, TseZ (BTH_II1884), which cooperates with $\mathrm{HmuR}$, the outer membrane heme transporter for zinc acquisition. T6SS secreted TseZ directly binds zinc ions and interacts with the heme transporter HmuR to transport zinc across the outer membrane. HmuR is a redox-regulated dual functional transporter. Under normal conditions, $\mathrm{HmuR}$ is used mainly for the transport of heme-iron; HmuR switches to transport of zinc upon sensing extracellular oxidative stress. Under mild oxidative stress condition, HmuR-mediated zinc transport alone is sufficient to maintain intracellular redox homeostasis. In contrast, under severe oxidative stress challenge, T6SS-4 is fully induced and secretes the proteinaceous zincophore TseZ to enhance the efficiency of HmuR-mediated zinc transport (Si et al., 2017a).

In Burkholderia pseudomallei, the T6SS-2 gene cluster also encodes a zinc binding protein (TseZ). TonB-dependent transporters that interact with Tse $\mathrm{Z}$ and actively transport $\mathrm{Zn}^{2+}$ across the outer membrane have also been identified as BhuR (DeShazer, 2019).

\section{Manganese}

Manganese is also an essential micronutrient for many cellular components or processes, such as lipid, protein, carbohydrate metabolism, transcriptional regulation, and resistance to oxidative stress (Kehres and Maguire, 2003). Manganese plays a crucial role in bacterial iron homeostasis and protection against oxidative damage (Puri et al., 2010). Two manganese ions, $\mathrm{Mn}^{2+}$ and $\mathrm{Mn}^{3+}$, are found in most organisms. In contrast to $\mathrm{Fe}^{2+}$, free $\mathrm{Mn}^{2+}$ is not toxic in a biological environment; thus, it can replace the more reactive $\mathrm{Fe}^{2+}$ in $\mathrm{Fe}^{2+}$-containing proteins, reducing oxidative damage to 
these proteins (Hood and Skaar, 2012). Manganese can also enhance oxidative stress resistance by serving as a cofactor for ROS-detoxifying enzymes, such as SodA and KatN, or through the formation of non-proteinaceous manganese antioxidants (Aguirre and Culotta, 2012; Barnese et al., 2012). Two major transporters import extracellular manganese across the cytoplasmic membrane: a proton-dependent Nramprelated transport system $(\mathrm{MntH})$ and an ATP-binding cassette transporter (SitABCD and YfeABCD) (Goswami et al., 2001; Forbes and Gros, 2003).

The B. thailandensis T6SS-4 plays an important role in survival under oxidative stress by uptake $\mathrm{Mn}^{2+}$ through secreting TseM (BTH_II1883). TseM, a T6SS-4-dependent $\mathrm{Mn}^{2+}$-binding effector, is involved in the intracellular accumulation of manganese $\left(\mathrm{Mn}^{2+}\right)$ under oxidative stress, and an $\mathrm{Mn}^{2+}$-specific TonB-dependent outer membrane transporter MnoT, has been shown to be its interacting partner (Si et al., 2017b). Under high $\mathrm{Mn}^{2+}$ conditions, passive diffusion of $\mathrm{Mn}^{2+}$ through porins fulfills cellular $\mathrm{Mn}^{2+}$ requirements. Low $\mathrm{Mn}^{2+}$ triggers the induction of the TonB-dependent outer membrane transporter MnoT for the active transport of $\mathrm{Mn}^{2+}$ across the outer membrane. T6SS-4 expression is activated by the conserved oxidative stress regulator OxyR. Activated T6SS-4 secretes TseM into the extracellular milieu to scavenge $\mathrm{Mn}^{2+}$ and delivers its $\mathrm{Mn}^{2+}$ load to MnoT via direct interaction. The T6SS-MnoT mediated active $\mathrm{Mn}^{2+}$ transport system also participates in the interbacterial competition and bacterial virulence. The T6SS4 provides growth advantage in nutrient-limited environments and is critical for virulence in Galleria mellonella larvae ( $\mathrm{Si}$ et al., 2017b). Similarly, a $\mathrm{Mn}^{2+}$-binding effector (TseM) secreted by T6SS-2, together with its transmembrane transporter MnoT, was used to maintain redox homeostasis via $\mathrm{Mn}^{2+}$ acquisition in the B. pseudomallei complex (DeShazer, 2019). Recently, Y. pseudotuberculosis T6SS-4 was also found to secret a $\mathrm{Mn}^{2+}$-binding micropeptide, TssS, for $\mathrm{Mn}^{2+}$ acquisition and oxidative stress resistance. Remarkably, TssS was revealed to be delivered into host cells to inhibit the STING-mediated innate immune response by sequestering $\mathrm{Mn}^{2+}$. This finding provides a new perspective on the role of the T6SS in pathogenesis (Zhu et al., 2021).

\section{Iron}

Iron is an essential nutrient for living organisms by acting as a cofactor for a large number of enzymes and regulatory proteins. Although iron is abundant in the Earth's crust, the bioavailability iron is severely restricted due to extremely low solubility under aerobic conditions (Schaible and Kaufmann, 2004; Miethke and Marahiel, 2007). To acquire sufficient iron for growth, bacteria have evolved several strategies, including import of ferrous iron by ATP- or GTP-dependent inner membrane transporters and TonB-ExbB-ExbD-dependent transport of ferric-siderophores, transferrin, haem, or haem-bound proteins through specific outer membrane receptors (Braun, 2001; Hood and Skaar, 2012).

Pseudomonas aeruginosa competes for iron by producing the high affinity siderophores pyoverdine and pyochelin, as well as hemophores, and it can also import xenosiderophores released by other bacteria (Cornelis, 2010). As a P. aeruginosa mutant lacking three known iron acquisition systems (PA $\Delta 3 \mathrm{Fe}$ ) retains the ability to grow in an iron deficient media, a novel iron acquisition pathway coupling the H3-T6SS effector TseF (PA2374), Pseudomonas quinolone signal (PQS, 2-heptyl-3hydroxy-4-quinolone), outer membrane vesicles (OMVs), and the outer membrane receptors FptA and OprF was identified (Lin et al., 2017). TseF does not bind iron, but it interacts with the iron chelating PQS with a high affinity. The PQS molecule has been long known to bind iron with a high affinity but the physiological role of such binding remains unknown (Bredenbruch et al., 2006; Diggle et al., 2006). TseF engages siderophore receptor FptA and the porin OprF for iron acquisition (Nissen-Meyer et al., 1992). Consistent with the biochemical results, both FptA and OprF are required for TseF-mediated iron acquisition. Like the hydrophobic PQS, TseF is incorporated into outer membrane vesicles (OMVs), which have been suggested to play a role in iron acquisition in $P$. aeruginosa by unknown mechanism (Kulp and Kuehn, 2010). The T6SS substrate TseF integrates several molecules previously known to be involved in iron acquisition to transport iron to the cell. The $t s e F$ gene is present in many bacteria, suggesting wide use of this iron acquisition mechanism. The H3-T6SSpromoters and tseF expression for iron acquisition are commonly repressed by the ferric uptake regulator (Fur) for intracellular iron homeostasis (Lin et al., 2017). In Cupriavidus necator, T6SS1 secreted TeoL preferentially in association with OMVs through interactions with LPS, which enables bacterial cells to recruit OMVs derived from different species and confers advantages to bacterial cells for iron acquisition (Li et al., 2021).

An iron chelator, pyoverdine, secreted by Pseudomonas taiwanensis, can inhibit the growth of the rice bacterial blight pathogen Xanthomonas oryzae pv. oryzae (Xoo). T6SS is involved in the secretion of the endogenous iron chelator pyoverdine; however, the mechanism is unknown (Chen et al., 2016). Notably, the regulation of T6SS by Fur or iron has also been reported in Escherichia coli (Brunet et al., 2011), Edwardsiella tarda (Chakraborty et al., 2011), Burkholderia mallei, and B. pseudomallei (Burtnick and Brett, 2013), implicating the possible roles of these T6SSs in iron acquisition.

\section{Copper}

As one of the most stable divalent transition metals, cupric copper $\left(\mathrm{Cu}^{2+}\right)$ displays a high affinity for metalloproteins (Waldron and Robinson, 2009). Copper is a catalyzer for electron transfer reactions in bacteria and a cofactor of copper-detoxifying enzymes (Dupont et al., 2011; Hodgkinson and Petris, 2012). Because copper is toxic, intracellular copper levels must be tightly controlled to ensure the homeostasis required for cuproprotein synthesis and prevent toxic effects (Argüello et al., 2013). ComC in $E$. coli represses copper uptake and thus plays an important role in copper homeostasis, and its homologs have been found in many gram-negative bacteria (Rademacher and Masepohl, 2012). For the import of copper, a few cytoplasmic $\mathrm{Cu}^{2+}$ sensing transcriptional regulators (CueR, CsoR, and CopY) (Strausak and Solioz, 1997; Outten et al., 2000; Liu et al., 2007) and periplasmic $\mathrm{Cu}^{2+}$-sensing two-component systems (CopR/S, CusR/S, and PcoR/S) (Rensing and Grass, 2003; Teitzel et al., 2006) have been found to play important roles. However, 
copper efflux in pathogenic enterobacteria is more crucial than copper uptake (Nies and Herzberg, 2013). The inner membrane heavy metal pumps (transmembrane P1B-type ATPases) in many gram-negative bacteria are responsible for the exportation of cytoplasmic copper to the periplasm (Klein and Lewinson, 2011).

In $P$. aeruginosa, azurin (Azu) has a high affinity for oxidized $\mathrm{Cu}^{2+}$-bound proteins (Nar et al., 1992; Zhang and Rainey, 2008). Based on an analysis of the $P$. aeruginosa H2-T6SS-dependent secretomes, Azu was characterized as an H2-T6SS-dependent copper $\left(\mathrm{Cu}^{2+}\right)$-binding effector. OprC, a $\mathrm{Cu}^{2+}$-specific TonBdependent outer membrane transporter, has been identified as an Azu-interacting partner. Both $\mathrm{Azu}$ and $\mathrm{OprC}$ are directly regulated by the transcriptional regulator $\mathrm{CueR}$ and are induced by low $\mathrm{Cu}^{2+}$ concentrations (Han et al., 2019).

Pseudomonas aeruginosa possesses three T6SS loci: H1-, H2-, and H3-T6SSs that provide a fitness advantage in bacterial community competition by delivering toxins to target cells (Mougous et al., 2006; Russell et al., 2011). The identified T6SS-dependent antibacterial toxin effectors include Tse1-Tse3, PldA, TplE, and PldB (Russell et al., 2011; Jiang et al., 2014; Sana et al., 2015). T6SS-mediated $\mathrm{Cu}$ acquisition also provides a growth advantage in bacterial competition, indicating the critical role of the Azu-OprC-mediated $\mathrm{Cu}^{2+}$ transport system (Han et al., 2019). Like VgrG2b that is secreted by H2-T6SS and shows an anti-eukaryotic function, the H2-T6SS-dependent $\mathrm{Cu}^{2+}$ transport system is important for bacterial virulence in the blood and lungs of infected mice (Sana et al., 2015; Han et al., 2019).

\section{Molybdenum}

Molybdenum is a trace metal element for nitrate metabolism in many bacteria and exists in the form of its oxyanion, molybdate $\left(\mathrm{MoO}_{4}{ }^{2-}\right)$ under natural conditions (Grunden and Shanmugam, 1997). Bacteria acquire molybdate mainly through the highaffinity ATP-binding cassette permease ModABC (Pederick et al., 2014) and non-specific anion importers (Self et al., 2001). The imported $\mathrm{MoO}_{4}{ }^{2-}$ often becomes a part of the Manganese chelating protein molecule to form a molybdenum cofactor, participating in the activity of molybdo-enzymes (Kraft et al., 2011).

In $P$. aeruginosa, the H2-T6SS secreted ModA has been identified as a molybdate-binding protein and mediated molybdate acquisition. Moreover, a ModA partner that participates in molybdate transport has also been identified as $\mathrm{IcmP}$ that is an insulin-cleaving metalloproteinase outer membrane protein (Wang et al., 2021). The T6SS-ModA-IcmP system contributes to bacterial virulence and participates in bacterial competition under anaerobic conditions. Studies have shown that the molybdenum homeostasis of $P$. aeruginosa PA1006 is necessary for nitrate utilization, biofilm formation, and virulence (Filiatrault et al., 2013; Tombline et al., 2013). In a mouse model of acute pneumonia, the P. aeruginosa $\Delta c l p V 2$,

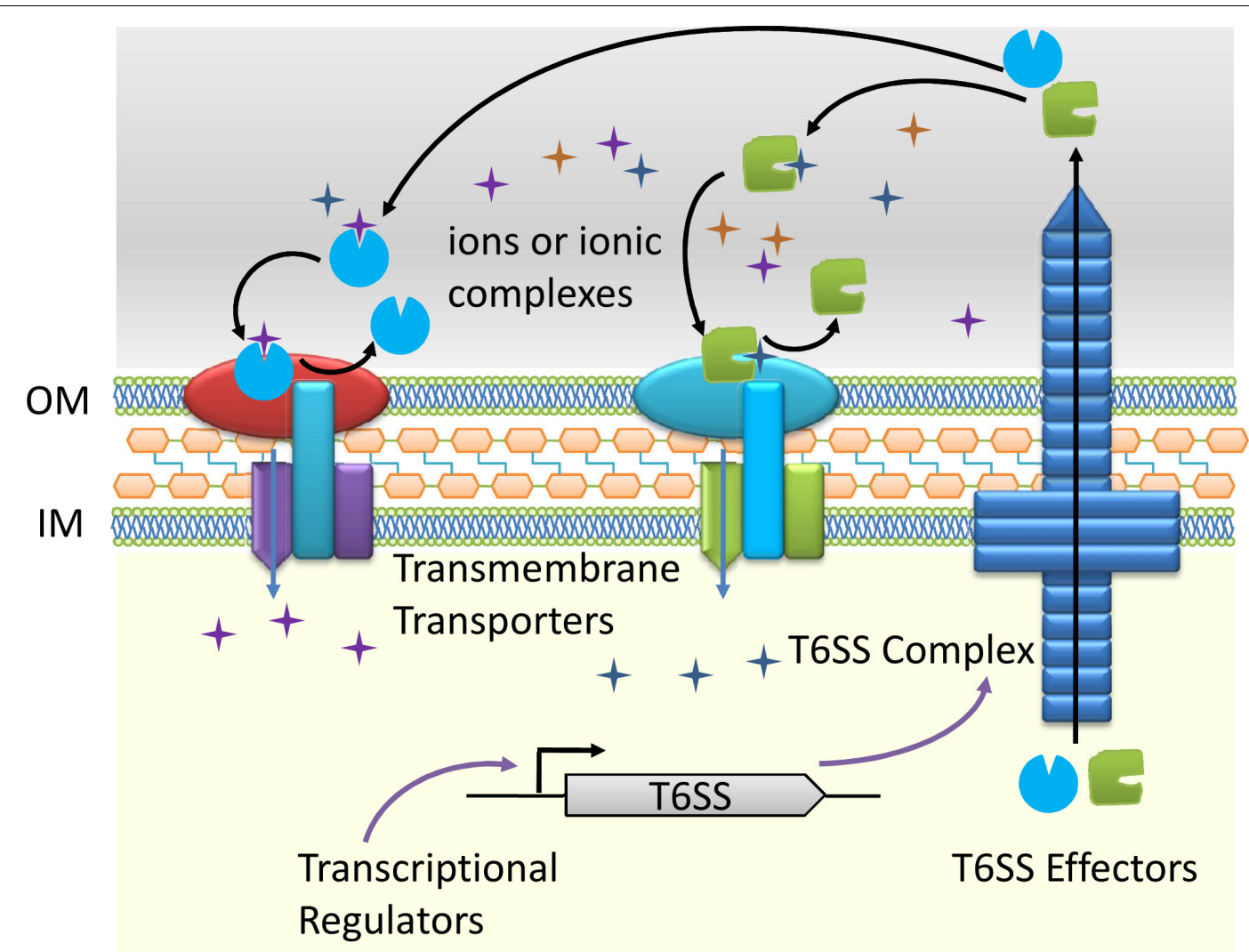

FIGURE 1 | Schematic diagram of the type VI secretion system (T6SS) dependent ions transport. 
$\Delta \bmod A$, and $\Delta a n r$ mutants exhibited attenuated virulence, indicating that the H2-T6SS-mediated molybdate transport system contributes to the resistance to host nutritional immunity (Wang et al., 2021). Like the previously discovered two H1-T6SS effectors Tse 1 and Tse3, which can hydrolyze peptidoglycan and provide a competitive fitness advantage (Russell et al., 2011), H2T6SS improves bacterial competition by promoting molybdate $\left(\mathrm{MoO}_{4}{ }^{2-}\right.$ ) acquisition under anaerobic conditions (Wang et al., 2021). As a transcriptional regulator, Anr can activate H2-T6SS expression under anaerobic conditions (Wang et al., 2021). Anr in $P$. aeruginosa controls the switch from aerobic to anaerobic growth and plays a pivotal role in adapting to microaerobic or anoxic conditions (Ugidos et al., 2008; Tata et al., 2017).

\section{CONCLUSION}

All organisms keep metal homeostasis for physiological demands by sensing small fluctuations in metal levels (Porcheron et al., 2013). Bacteria have developed complex transport systems for each metal whose expression is coordinated by their corresponding regulators (such as Fur, MntR, CueR, and Zur, etc.) (Wakeman and Skaar, 2012). T6SS was a newly found device for bacteria to acquire metal ions, expanding our understanding on sophistication of bacterial metal ion acquisition systems. T6SS participation in metal ion uptake, which assists the bacterial low- and high-affinity transport systems to scavenge from the environment essential metals in chelated or free forms. In addition, the metal ion transport function of T6SS is usually involved in multiple biological processes and is crucial for bacterial survival and host colonization. Traditionally, T6SS is recognized as a contact-dependent molecular machinery. Recent studies revealed that T6SSs play crucial roles in shaping the composition of a microbial population in hosts or environmental niches, either by directly killing competing cells via contactdependent (Russell et al., 2011) and contact-independent (Song et al., 2021) translocation of toxins, or by competing for essential nutrients via contact-independent secretion of metal ion binding

\section{REFERENCES}

Aguirre, J. D., and Culotta, V. C. (2012). Battles with iron: manganese in oxidative stress protection. J. Biol. Chem. 287, 13541-13548. doi: 10.1074/jbc.R111. 312181

Andreini, C., Banci, L., Bertini, I., and Rosato, A. (2006). Zinc through the three domains of life. J. Proteome Res. 5, 3173-3178. doi: 10.1021/pr0603699

Argüello, J. M., Raimunda, D., and Padilla-Benavides, T. (2013). Mechanisms of copper homeostasis in bacteria. Front. Cell Infect. Microbiol. 3:73. doi: 10.3389/ fcimb.2013.00073

Barnese, K., Gralla, E. B., Valentine, J. S., and Cabelli, D. E. (2012). Biologically relevant mechanism for catalytic superoxide removal by simple manganese compounds. Proc. Natl. Acad. Sci. U. S. A. 109:6892. doi: 10.1073/pnas. 1203051109

Borgeaud, S., Metzger, L. C., Scrignari, T., and Blokesch, M. (2015). The type VI secretion system of Vibrio cholerae fosters horizontal gene transfer. Science 347, 63-67. doi: 10.1126/science. 1260064

Braun, V. (2001). Iron uptake mechanisms and their regulation in pathogenic bacteria. Int. J. Med. Microbiol. 291, 67-79. doi: 10.1078/1438-4221-00103

Bredenbruch, F., Geffers, R., Nimtz, M., Buer, J., and Haussler, S. (2006). The Pseudomonas aeruginosa quinolone signal (PQS) has an iron-chelating activity. Environ. Microbiol. 8, 1318-1329. doi: 10.1111/j.1462-2920.2006.01025.x effectors. For example, the T6SS-HmuR-mediated active zinc transport system is involved in a contact-independent bacteriabacteria competition for nutrients (Si et al., 2017a,b; Han et al., 2019; Wang et al., 2021).

A schematic diagram was used to show the process of metal ions transport through T6SS (Figure 1). Briefly, the T6SS dependent effectors bind to specific metal ions or ionic complexes in the environment. Accompanied by a transmembrane ion transporter, the corresponding ions are transferred to the cell. The ions transport process is collaboratively fulfilled by the T6SS effectors and their transmembrane partners. It is worth mentioning that the metal ions transporting function of T6SS is often activated under special circumstances, such as low ions concentrations or environmental stresses and is regulated by multiple transcriptional regulators. So far, it is unknown whether T6SS correlates with metal ions efflux. We believe that ions transport through T6SS expands the range of functions associated with this secretory nanomachines and merits additional studies in other bacteria.

\section{AUTHOR CONTRIBUTIONS}

$\mathrm{XY}, \mathrm{HL}$, and YZ collected and assessed the references. XS and $\mathrm{HL}$ contributed in the proposal and article layout of the review. $\mathrm{XY}$ and XS wrote the manuscript. All authors contributed to the article and approved the submitted version.

\section{FUNDING}

This work was supported by the grant of National Key R\&D Program of China (2018YFA0901200), National Natural Science Foundation of China (31725003 and 31670053), and Comprehensive treatment of Xiaokongtong Gully Project: Research and application of in situ greening and soil consolidation technology (QYLF-JSYY-2020029).

Brunet, Y. R., Bernard, C. S., Gavioli, M., Lloubes, R., and Cascales, E. (2011). An epigenetic switch involving overlapping fur and DNA methylation optimizes expression of a type VI secretion gene cluster. PLoS Genet. 7:e1002205. doi: 10.1371/journal.pgen.1002205

Burtnick, M. N., and Brett, P. J. (2013). Burkholderia mallei and Burkholderia pseudomallei cluster 1 type VI secretion system gene expression is negatively regulated by iron and zinc. PLoS One 8:e76767. doi: 10.1371/journal.pone. 0076767

Cai, R., Gao, F., Pan, J., Hao, X., Yu, Z., and Qu, Y. (2021). The transcriptional regulator Zur regulates the expression of ZnuABC and T6SS4 in response to stresses in Yersinia pseudotuberculosis. Microbiol. Res. 249:126787. doi: 10.1016/ j.micres.2021.126787

Chakraborty, S., Sivaraman, J., Leung, K. Y., and Mok, Y. K. (2011). Twocomponent $\mathrm{PhoB}-\mathrm{PhoR}$ regulatory system and ferric uptake regulator sense phosphate and iron to control virulence genes in type III and VI secretion systems of Edwardsiella tarda. J. Biol. Chem. 286, 39417-39430. doi: 10.1074/ jbc.M111.295188

Chassaing, B., and Cascales, E. (2018). Antibacterial Weapons: targeted Destruction in the Microbiota. Trends Microbiol. 26, 329-338. doi: 10.1016/ j.tim.2018.01.006

Chen, W. J., Kuo, T. Y., Hsieh, F. C., Chen, P. Y., Wang, C. S., Shih, Y. L., et al. (2016). Involvement of type VI secretion system in secretion of iron 
chelator pyoverdine in Pseudomonas taiwanensis. Sci. Rep. 6:32950. doi: 10. 1038/srep32950

Chow, J., and Mazmanian, S. K. (2010). A pathobiont of the microbiota balances host colonization and intestinal inflammation. Cell Host Microbe 7, 265-276. doi: 10.1016/j.chom.2010.03.004

Cianfanelli, F. R., Monlezun, L., and Coulthurst, S. J. (2016). Aim, Load, Fire: the Type VI Secretion System, a Bacterial Nanoweapon. Trends Microbiol. 24, 51-62. doi: 10.1016/j.tim.2015.10.005

Cornelis, P. (2010). Iron uptake and metabolism in pseudomonads. Appl. Microbiol. Biotechnol. 86, 1637-1645. doi: 10.1007/s00253-010-2550-2

Coulthurst, S. (2019). The Type VI secretion system: a versatile bacterial weapon. Microbiology 165, 503-515. doi: 10.1099/mic.0.000789

DeShazer, D. (2019). A novel contact-independent T6SS that maintains redox homeostasis via $\mathrm{Zn}^{2+}$ and $\mathrm{Mn}^{2+}$ acquisition is conserved in the Burkholderia pseudomallei complex. Microbiol. Res. 226, 48-54. doi: 10.1016/j.micres.2019. 05.007

Diggle, S. P., Lumjiaktase, P., Dipilato, F., Winzer, K., Kunakorn, M., Barrett, D. A., et al. (2006). Functional genetic analysis reveals a 2-Alkyl-4-quinolone signaling system in the human pathogen Burkholderia pseudomallei and related bacteria. Chem. Biol. 13, 701-710. doi: 10.1016/j.chembiol.2006.05.006

Dupont, C. L., Grass, G., and Rensing, C. (2011). Copper toxicity and the origin of bacterial resistance-new insights and applications. Metallomics 3, 1109-1118.

Filiatrault, M. J., Tombline, G., Wagner, V. E., Van Alst, N., Rumbaugh, K., Sokol, P., et al. (2013). Pseudomonas aeruginosa PA1006, which plays a role in molybdenum homeostasis, is required for nitrate utilization, biofilm formation, and virulence. PLoS One 8:e55594. doi: 10.1371/journal.pone.0055594

Forbes, J. R., and Gros, P. (2003). Iron, manganese, and cobalt transport by Nramp1 (Slc11a1) and Nramp2 (Slc11a2) expressed at the plasma membrane. Blood 102, 1884-1892. doi: 10.1182/blood-2003-02-0425

Gallique, M., Decoin, V., Barbey, C., Rosay, T., Feuilloley, M. G., Orange, N., et al. (2017). Contribution of the Pseudomonas fluorescens MFE01 Type VI Secretion System to Biofilm Formation. PLoS One 12:e0170770. doi: 10.1371/ journal.pone. 0170770

Goswami, T., Bhattacharjee, A., Babal, P., Searle, S., and Moore, E. (2001). Naturalresistance-associated macrophage protein 1 is an $\mathrm{H}+$ /bivalent cation antiporter. Biochem. J. 354, 511-519. doi: 10.1042/bj3540511

Grunden, A. M., and Shanmugam, K. T. (1997). Molybdate transport and regulation in bacteria. Arch. Microbiol. 168, 345-354. doi: 10.1007/ s002030050508

Guan, J., Xiao, X., Xu, S., Gao, F., and Wang, J. (2015). Roles of RpoS in Yersinia pseudotuberculosis stress survival, motility, biofilm formation and type VI secretion system expression. J. Microbiol. 53, 633-642. doi: 10.1007/s12275015-0099-6

Gueguen, E., Durand, E., Zhang, X. Y., d'Amalric, Q., Journet, L., and Cascales, E. (2013). Expression of a Yersinia pseudotuberculosis Type VI Secretion System Is Responsive to Envelope Stresses through the OmpR Transcriptional Activator. PLoS One 8:e66615. doi: 10.1371/journal.pone.0066615

Han, Y., Wang, T., Chen, G., Pu, Q., Liu, Q., Zhang, Y., et al. (2019). A Pseudomonas aeruginosa type VI secretion system regulated by CueR facilitates copper acquisition. PLoS Pathog. 15:e1008198. doi: 10.1371/journal.ppat.1008198

Hantke, K. (2005). Bacterial zinc uptake and regulators. Curr. Opin. Microbiol. 8, 196-202. doi: 10.1016/j.mib.2005.02.001

Hodgkinson, V., and Petris, M. J. (2012). Copper homeostasis at the host-pathogen interface. J. Biol. Chem. 287, 13549-13555. doi: 10.1074/jbc.R111.316406

Hood, M. I., Mortensen, B. L., Moore, J. L., Zhang, Y., Kehl-Fie, T. E., Sugitani, N., et al. (2012). Identification of an Acinetobacter baumannii zinc acquisition system that facilitates resistance to calprotectin-mediated zinc sequestration. PLoS Pathog. 8:e1003068. doi: 10.1371/journal.ppat.1003068

Hood, M. I., and Skaar, E. P. (2012). Nutritional immunity: transition metals at the pathogen-host interface. Nat. Rev. Microbiol. 10, 525-537. doi: 10.1038/ nrmicro2836

Hood, R. D., Singh, P., Hsu, F., Guvener, T., and Carl, M. A. (2010). A type VI secretion system of Pseudomonas aeruginosa targets a toxin to bacteria. Cell Host Microbe 7, 25-37. doi: 10.1016/j.chom.2009.12.007

Isohanni, P., Huehn, S., Aho, T., Alter, T., and Lyhs, U. (2013). Heat stress adaptation induces cross-protection against lethal acid stress conditions in Arcobacter butzleri but not in Campylobacter jejuni. Food Microbiol. 34, 431435. doi: 10.1016/j.fm.2013.02.001
Jiang, F., Waterfield, N. R., Yang, J., Yang, G., and Jin, Q. (2014). A Pseudomonas aeruginosa type VI secretion phospholipase $\mathrm{D}$ effector targets both prokaryotic and eukaryotic cells. Cell Host Microbe 15, 600-610. doi: 10.1016/j.chom.2014. 04.010

Kehres, D. G., and Maguire, M. E. (2003). Emerging themes in manganese transport, biochemistry and pathogenesis in bacteria. FEMS Microbiol. Rev. 27, 263-290. doi: 10.1016/S0168-6445(03)00052-4

Klein, J. S., and Lewinson, O. (2011). Bacterial ATP-driven transporters of transition metals: physiological roles, mechanisms of action, and roles in bacterial virulence. Metallomics 3, 1098-1108. doi: 10.1039/c1mt00073j

Kraft, B., Strous, M., and Tegetmeyer, H. E. (2011). Microbial nitrate respirationgenes, enzymes and environmental distribution. J. Biotechnol. 155, 104-117. doi: $10.1016 /$ j.jbiotec.2010.12.025

Kulp, A., and Kuehn, M. J. (2010). Biological functions and biogenesis of secreted bacterial outer membrane vesicles. Annu. Rev. Microbiol. 64, 163-184. doi: 10.1146/annurev.micro.091208.073413

Li, C., Zhu, L., Wang, D., Wei, Z., Hao, X., Wang, Z., et al. (2021). T6SS secretes an LPS-binding effector to recruit OMVs for exploitative competition and horizontal gene transfer. ISME J. doi: 10.1038/s41396-021-01093-8 [Online ahead of print].

Lin, J., Zhang, W., Cheng, J., Yang, X., Zhu, K., Wang, Y., et al. (2017). A Pseudomonas T6SS effector recruits PQS-containing outer membrane vesicles for iron acquisition. Nat. Commun. 8:14888. doi: 10.1038/ncomms14888

Liu, J. Z., Jellbauer, S., Poe, A. J., Ton, V., Pesciaroli, M., and Kehl-Fie, T. E. (2012). Zinc Sequestration by the Neutrophil Protein Calprotect in Enhances Salmonella Growth in the Inflamed Gut. Cell Host Microbe 11, 227-239. doi: 10.1016/j.chom.2012.01.017

Liu, T., Ramesh, A., Ma, Z., Ward, S. K., Zhang, L., George, G. N., et al. (2007). CsoR is a novel Mycobacterium tuberculosis copper-sensing transcriptional regulator. Nat. Chem. Biol. 3, 60-68. doi: 10.1038/nchembio844

Miethke, M., and Marahiel, M. A. (2007). Siderophore-based iron acquisition and pathogen control. Microbiol. Mol. Biol. Rev. 71, 413-451.

Mols, M., and Abee, T. (2011). Primary and secondary oxidative stress in Bacillus. Environ. Microbiol. 13, 1387-1394. doi: 10.1111/j.1462-2920.2011. 02433.x

Mougous, J. D., Cuff, M. E., Raunser, S., Shen, A., Zhou, M., Gifford, C. A., et al. (2006). A virulence locus of Pseudomonas aeruginosa encodes a protein secretion apparatus. Science 312, 1526-1530. doi: 10.1126/science.112 8393

Nar, H., Messerschmidt, A., Huber, R., van de Kamp, M., and Canters, G. W. (1992). Crystal structure of Pseudomonas aeruginosa apo-azurin at 1.85 A resolution. FEBS Lett. 306, 119-124. doi: 10.1016/0014-5793(92)80981-L

Nies, D. H., and Herzberg, M. (2013). A fresh view of the cell biology of copper in enterobacteria. Mol. Microbiol. 87, 447-454. doi: 10.1111/mmi.12123

Nissen-Meyer, J., Holo, H., Håvarstein, L. S., Sletten, K., and Nes, I. F. (1992). A novel lactococcal bacteriocin whose activity depends on the complementary action of two peptides. J. Bacteriol. 174, 5686-5692.

Outten, F. W., Outten, C. E., Hale, J., and O'Halloran, T. V. (2000). Transcriptional activation of an Escherichia coli copper efflux regulon by the chromosomal MerR homologue, cueR. J. Biol. Chem. 275, 31024-31029. doi: 10.1074/jbc. M006508200

Pederick, V. G., Eijkelkamp, B. A., Ween, M. P., Begg, S. L., Paton, J. C., and McDevitt, C. A. (2014). Acquisition and role of molybdate in Pseudomonas aeruginosa. Appl. Environ. Microbiol. 80, 6843-6852.

Porcheron, G., Garenaux, A., Proulx, J., Sabri, M., and Dozois, C. M. (2013). Iron, copper, zinc, and manganese transport and regulation in pathogenic Enterobacteria: correlations between strains, site of infection and the relative importance of the different metal transport systems for virulence. Front. Cell Infect. Microbiol. 3:90. doi: 10.3389/fcimb.2013.00090

Pukatzki, S., Ma, A. T., Sturtevant, D., Krastins, B., Sarracino, D., Nelson, W. C., et al. (2006). Identification of a conserved bacterial protein secretion system in Vibrio cholerae using the Dictyostelium host model system. Proc. Natl. Acad. Sci. U. S. A. 103, 1528-1533. doi: 10.1073/pnas.0510322103

Puri, S., Hohle, T. H., and O'Brian, M. R. (2010). Control of bacterial iron homeostasis by manganese. Proc. Natl. Acad. Sci. U. S. A. 107, 10691-10695. doi: 10.1073/pnas.1002342107

Rademacher, C., and Masepohl, B. (2012). Copper-responsive gene regulation in bacteria. Microbiology 158, 2451-2464. doi: 10.1099/mic.0.058487-0 
Rensing, C., and Grass, G. (2003). Escherichia coli mechanisms of copper homeostasis in a changing environment. FEMS Microbiol. Rev. 27, 197-213. doi: 10.1016/S0168-6445(03)00049-4

Russell, A. B., Hood, R. D., Bui, N. K., LeRoux, M., Vollmer, W., and Mougous, J. D. (2011). Type VI secretion delivers bacteriolytic effectors to target cells. Nature 475, 343-347. doi: 10.1038/nature10244

Sana, T. G., Baumann, C., Merdes, A., Soscia, C., Rattei, T., Hachani, A., et al. (2015). Internalization of Pseudomonas aeruginosa Strain PAO1 into Epithelial Cells Is Promoted by Interaction of a T6SS Effector with the Microtubule Network. mBio 6:e00712. doi: 10.1128/mBio.00712-15

Schaible, U. E., and Kaufmann, S. H. (2004). Iron and microbial infection. Nat. Rev. Microbiol. 2, 946-953. doi: 10.1038/nrmicro1046

Self, W. T., Grunden, A. M., Hasona, A., and Shanmugam, K. T. (2001). Molybdate transport. Res. Microbiol. 152, 311-321. doi: 10.1016/S0923-2508(01)01202-5

Si, M., Wang, Y., Zhang, B., Zhao, C., Kang, Y., Bai, H., et al. (2017a). The Type VI Secretion System Engages a Redox-Regulated Dual-Functional Heme Transporter for Zinc Acquisition. Cell Rep. 20, 949-959. doi: 10.1016/j.celrep. 2017.06.081

Si, M., Zhao, C., Burkinshaw, B., Zhang, B., Wei, D., Wang, Y., et al. (2017b). Manganese scavenging and oxidative stress response mediated by type VI secretion system in Burkholderia thailandensis. Proc. Natl. Acad. Sci. U. S. A. 114, E2233-E2242. doi: 10.1073/pnas.1614902114

Song, L., Pan, J., Yang, Y., Zhang, Z., and Cui, R. (2021). Contact-independent killing mediated by a T6SS effector with intrinsic cell-entry properties. Nat. Commun. 12:423. doi: 10.1038/s41467-020-20726-8

Song, Y., Xiao, X., Li, C., Wang, T., Zhao, R., Zhang, W., et al. (2015). The dual transcriptional regulator RovM regulates the expression of AR3- and T6SS4dependent acid survival systems in response to nutritional status in Yersinia pseudotuberculosis. Environ. Microbiol. 17, 4631-4645.

Strausak, D., and Solioz, M. (1997). CopY is a copper-inducible repressor of the Enterococcus hirae copper ATPases. J. Biol. Chem. 272, 8932-8936. doi: 10.1074/ jbc. 272.14 .8932

Tata, M., Amman, F., Pawar, V., Wolfinger, M. T., Weiss, S., Häussler, S., et al. (2017). The Anaerobically Induced sRNA Pail Affects Denitrification in Pseudomonas aeruginosa PA14. Front. Microbiol. 8:2312. doi: 10.3389/fmicb. 2017.02312

Teitzel, G. M., Geddie, A., De Long, S. K., Kirisits, M. J., Whiteley, M., Parsek, M. R., et al. (2006). Survival and growth in the presence of elevated copper: transcriptional profiling of copper-stressed Pseudomonas aeruginosa. J. Bacteriol. 188, 7242-7256. doi: 10.1128/JB.00837-06

Tombline, G., Schwingel, J. M., Lapek, J. D. Jr., Friedman, A. E., Darrah, T., Maguire, M., et al. (2013). Pseudomonas aeruginosa PA1006 is a persulfidemodified protein that is critical for molybdenum homeostasis. PLoS One 8:e55593. doi: 10.1371/journal.pone.0055593

Ugidos, A., Morales, G., Rial, E., Williams, H. D., and Rojo, F. (2008). The coordinate regulation of multiple terminal oxidases by the Pseudomonas putida ANR global regulator. Environ. Microbiol. 10, 1690-1702. doi: 10.1111/j.14622920.2008.01586.x

Wakeman, C. A., and Skaar, E. P. (2012). Metalloregulation of Gram-positive pathogen physiology. Curr. Opin. Microbiol. 15, 169-174. doi: 10.1016/j.mib. 2011.11.008

Waldron, K. J., and Robinson, N. J. (2009). How do bacterial cells ensure that metalloproteins get the correct metal?. Nat. Rev. Microbiol. 7, 25-35. doi: 10.1038/nrmicro2057

Wan, B., Zhang, Q., Ni, J., Li, S., Wen, D., Li, J., et al. (2017). Type VI secretion system contributes to Enterohemorrhagic Escherichia coli virulence by secreting catalase against host reactive oxygen species (ROS). PLoS Pathog. 13:e1006246. doi: 10.1371/journal.ppat.1006246

Wang, D., and Fierke, C. A. (2013). The BaeSR regulon is involved in defense against zinc toxicity in E. coli. Metallomics 5, 372-383. doi: 10.1039/ c3mt20217h
Wang, D., Hosteen, O., and Fierke, C. A. (2012). ZntR-mediated transcription of zntA responds to nanomolar intracellular free zinc. J. Inorg. Biochem. 111, 173-181. doi: 10.1016/j.jinorgbio.2012.02.008

Wang, T., Chen, K., Gao, F., Kang, Y., Chaudhry, M. T., Wang, Z., et al. (2017). ZntR positively regulates T6SS4 expression in Yersinia pseudotuberculosis. J. Microbiol. 55, 448-456. doi: 10.1007/s12275-017-6540-2

Wang, T., Du, X., Ji, L., Han, Y., Dang, J., Wen, J., et al. (2021). Pseudomonas aeruginosa T6SS-mediated molybdate transport contributes to bacterial competition during anaerobiosis. Cell Rep. 35:108957. doi: 10.1016/j.celrep. 2021.108957

Wang, T., Si, M., Song, Y., Zhu, W., Gao, F., Wang, Y., et al. (2015). Type VI Secretion System Transports $\mathrm{Zn}^{2+}$ to Combat Multiple Stresses and Host Immunity. PLoS Pathog. 11:e1005020. doi: 10.1371/journal.ppat.100 5020

Weber, B., Hasic, M., Chen, C., Wai, S. N., and Milton, D. L. (2009). Type VI secretion modulates quorum sensing and stress response in Vibrio anguillarum. Environ. Microbiol. 11, 3018-3028.

Yang, X., Long, M., and Shen, X. (2018). Effector(-)Immunity Pairs Provide the T6SS Nanomachine its Offensive and Defensive Capabilities. Molecules 23:1009.

Yang, X., Song, Y., Dai, Q., Zhang, H., Song, L., Wang, Z., et al. (2019). The stringent response factor, RelA, positively regulates T6SS4 expression through the RovM/RovA pathway in Yersinia pseudotuberculosis. Microbiol. Res. 220, 32-41.

Zhang, L., Hinz, A. J., Nadeau, J. P., and Mah, T. F. (2011). Pseudomonas aeruginosa tssC1 links type VI secretion and biofilm-specific antibiotic resistance. J. Bacteriol. 193, 5510-5513. doi: 10.1128/JB.002 $68-11$

Zhang, W., Wang, Y., Song, Y., Wang, T., and Xu, S. (2013). A type VI secretion system regulated by OmpR in Yersinia pseudotuberculosis functions to maintain intracellular pH homeostasis. Environ. Microbiol. 15, 557-569. doi: 10.1111/ 1462-2920.12005

Zhang, X. X., and Rainey, P. B. (2008). Regulation of copper homeostasis in Pseudomonas fluorescens SBW25. Environ. Microbiol. 10, 3284-3294. doi: 10. 1111/j.1462-2920.2008.01720.x

Zhao, R., Song, Y., Dai, Q., Kang, Y., and Pan, J. (2017). A starvation-induced regulator, RovM, acts as a switch for planktonic/biofilm state transition in Yersinia pseudotuberculosis. Sci. Rep. 7:639.

Zhu, L., Xu, L., Wang, C., Li, C., Li, M., Liu, Q., et al. (2021). T6SS translocates a micropeptide to suppress STING-mediated innate immunity by sequestering manganese. Proc. Natl. Acad. Sci. U. S. A. 118:e2103526118.

Conflict of Interest: HL and YZ was employed by the company Qingyang Longfeng Sponge City Construction Management \& Operation Co., Ltd.

The remaining authors declare that the research was conducted in the absence of any commercial or financial relationships that could be construed as a potential conflict of interest.

Publisher's Note: All claims expressed in this article are solely those of the authors and do not necessarily represent those of their affiliated organizations, or those of the publisher, the editors and the reviewers. Any product that may be evaluated in this article, or claim that may be made by its manufacturer, is not guaranteed or endorsed by the publisher.

Copyright (c) 2021 Yang, Liu, Zhang and Shen. This is an open-access article distributed under the terms of the Creative Commons Attribution License (CC BY). The use, distribution or reproduction in other forums is permitted, provided the original author(s) and the copyright owner(s) are credited and that the original publication in this journal is cited, in accordance with accepted academic practice. No use, distribution or reproduction is permitted which does not comply with these terms. 